\title{
A Role for the Striatum in Regret-related Choice Repetition
}

\author{
Antoinette Nicolle, Dominik R. Bach, Jon Driver, \\ and Raymond J. Dolan
}

\begin{abstract}
"Regret aversion" is proposed to explain a tendency to avoid future choices that have induced past regret. However, regret might also motivate us to repeat previous regret-related choices to make up for their previous selection, a behavior resembling "chasing" in the context of gambling. In the current experiment, we acquired fMRI brain data while participants placed monetary bets on repeated gambles. Behaviorally, participants showed a tendency to repeat previously regret-related choices (operationalized as those leading to an outcome worse than what might have been), an effect restricted to early runs of the task. At gamble
\end{abstract}

\section{INTRODUCTION}

Regret is a cognitively mediated, multidimensional emotion engendered by counterfactual thinking (Roese, 1994; Markman, Gavanski, Sherman, \& Mcmullen, 1993), involving cognitions of how an alternate reality might have been better under a different choice. Such regret requires a sense of personal blame or responsibility, and its occurrence can exert a considerable impact on future choice behavior (e.g., Mellers, 1999; Bell, 1982; Loomes \& Sugden, 1982). Economic and psychological research has provided models of how regret may bias future choices (e.g., Mellers, Schwartz, Ho, \& Ritov, 1997; Bell, 1982; Loomes \& Sugden, 1982; Savage, 1951). The dominant perspective here has been that anticipation of regret may bias future decision-making by encouraging choices that serve to minimize the future occurrence of regret (Savage, 1951).

This prototypical economic perspective contrasts with an emerging view that regret invokes cognitive regulatory strategies, such that we mentally reconstruct an event or its antecedents to make ourselves feel better about mistaken choices (Roese \& Olson, 2007; Zeelenberg \& Pieters, 2007). For example, on narrowly missing a bus, we may reduce feelings of regret with self-justifying thoughts such as "I couldn't have run any faster" or "I couldn't have known to leave home earlier." The distinction between the prototypical economic perspective versus such theories of cognitive regulation suggests that regret-related events might in principle have multiple behavioral impacts.

University College London outcome, we show a reduction in ventral striatal activity for regretrelated relative to relief-related outcomes. Critically, this modulation was only seen when subjects were responsible for the bet choice. Activity in dorsal striatum was associated with an influence of previous regret on participants' subsequent choices, which is evident in increased activity when regret-related choices were repeated, relative to avoided, on the next trial. Our findings indicate that regret can lead to choice repetition as if seeking to make up for our mistakes and in so doing may lead to subsequent chasing behavior.
Repeating a previously regret-related choice might allow the decision maker to make up for the prior mistake. Such a strategy invokes an optimistic prospect that a favorable outcome is likely to ensue the second time around, thereby reducing dissonance from a previously regretful event. Such behavior would seem at odds with a view that we minimize the likely occurrence of future regret. However, we have recently shown that decision makers will repeat a previously regret-related choice if this gives the chance of a better return (Nicolle, in preparation), a behavior we suggest reflects a strategy aimed at compensating for a previous mistake. It is unclear, however, whether any such behavior regulated the current experience of a regretrelated outcome or whether it reflects some higher order goal-directed behavior.

Apparent compensatory risk-seeking after regret invokes a possible link between regret-regulatory strategies on the one hand and the well-recognized role of "chasing" in problem gambling (Lesieur, 1984) on the other hand. In the latter, there is a continuation of gambling after a series of losses. It has also been suggested that the phenomenon of a "near miss," which depends on comparison of an obtained outcome with a close better counterfactual outcome, may encourage chasing in the context of gambling (Reid, 1986). Moreover, a chasing strategy may provide gamblers with a potential means of reducing feelings of regret (Loftus \& Loftus, 1983). Whether such a strategy involves elimination or regulation of the experienced regret is unclear. Here we explore the behavioral nature and underlying neuronal mechanisms of choice under uncertainty after regret-related outcomes in a task that explicitly manipulated 
experienced regret (operationalized as explained below) while holding anticipated regret constant.

Guided by the economic literature, we considered regret as resulting from the operationally regret-related nature of a received outcome, being proportional to how much better the outcome would have been from a different choice compared with the outcome actually obtained. For simplicity, we used a binary choice, binary outcome (win/loss) gambling task in which a specific level of operationalized objective regret (and one of relief, where the outcome is better than might have been) could be induced on each trial. Because our main question concerned behavioral and neuronal responses to these operationalized regretrelated outcomes, anticipated regret was held constant across the two gamble choices. Either of the two possible bets (10p and 50p), made with the aim of winning the corresponding extra amount, could result in an outcome that could have been better from the alternative bet choice (in the form of $10 p$ wins or 50p losses). Using fMRI, we predicted that activity in ventral striatum, a region that encodes both passive and action-contingent rewards (O'Doherty et al., 2004), should be attenuated for regretrelated outcomes compared with relief-related outcomes. Critically, we also predicted that any such reduction should be dependent on participants being responsible for the bet selection (i.e., having agency; see also Coricelli et al., 2005). Although there has been some debate on whether outcome regret can exist in the absence of responsibility or agency (for a thorough review and discussion, see Ordonez \& Connolly, 2000; Zeelenberg, 2000; Zeelenberg, van Dijk, Manstead, \& van der Pligt, 1998; Connolly, 1997), behavioral and fMRI findings indicate that agency at least amplifies regret if not being necessary for its induction (see previously mentioned reviews and Coricelli et al., 2005). On the basis of prior findings of OFC involvement in regret (Coricelli et al., 2005), we also anticipated that activity here might show an influence of regret-related outcomes.

To address the possibility of a specific motivational impact of (our operationally defined) regret on choice repetition, we explored any tendency for participants to repeat bets that on the immediately preceding trial had resulted in a regret-related compared with a relief-related outcome. In a related manner, we specifically tested, via fMRI, for brain activity that would distinguish choices made following a previously regret-related outcome from choices following a previously relief-related outcome. On the basis of evidence for a dorsal-ventral dissociation in the roles of the striatum in reward learning and goal-directed action (for a review, see Wickens, Budd, Hyland, \& Arbuthnott, 2007), we predicted regional variations in the involvement of the striatum for the tendency to repeat previously regretrelated choices specifically in particular (unlike the simple response to the regret-related outcome, regardless of such repetition considerations). We suggest that such a functional-anatomical dissociation might allow independent processing of more "emotional" responses versus be- havioral responses to regret-related events, thus permitting selection of future actions that (although not always being a conventionally regret-averse response) may nonetheless bring the decision maker toward higher order goals, such as justifying our past actions by backing the same bet repeatedly in the hope that previous poor outcomes will not be repeated-analogously to gamble chasing. Because such a behavioral strategy would be in apparent conflict with the relatively decreased value of the regret-related outcome, we also tested for conflict-related activity in the ACC when such choices are made, a region commonly involved in the monitoring of conflict (Botvinick, Braver, Barch, Carter, \& Cohen, 2001) as well as being involved in choice switching versus sticking (Critchley et al., 2003; Bush et al., 2002).

\section{METHODS \\ Participants and Design}

Trials were ascribed to four categories in a $2 \times 2$ repeated measures design that was conditionalized on the outcome of the previous trial for the behavioral analysis. For the fMRI analysis, onsets were modeled at the outcome of each trial, and were separated according to the outcome of the current trial. The two factors were outcome (win or loss) and stake (high 50p or low 10p). In addition, by including our no-agency control (see below), we could also explore how any tendency to repeat the same choice after each outcome type interacted with agency (in a $2 \times 2 \times$ 2 design, now with the additional factor of agency vs. no agency). We recruited 20 participants (10 women) to take part in the experiment. All were right-handed with normal or corrected-to-normal vision by self-report and no history of neurological or psychiatric disorder. Participants were aged between 20 and 38 years (mean $=25.6$ years), and each gave informed consent in accord with procedures approved by the University College London Research Ethics Committee. Three participants were removed from the fMRI analysis because of faulty T1 images and resulting problems with image normalization, but those participants were included in the behavioral analysis for completeness.

\section{Experimental Procedure}

Participants were given an initial endowment of $£ 10$ and subsequently performed a gambling task in which they placed high (50p) or low (10p) bets on uncertain gambles. Participants were not informed of the relative probabilities of winning versus losing on each gamble, although these were in fact fixed at 50\%. Participants were each presented with a computer-simulated pack of red and blue cards turned facedown and were informed that the top card would be overturned on each trial. After placing their bet at the start of each trial, participants received a binary outcome of either a win (if the card was blue, as on $50 \%$ of trials) or a loss (if it was red, as on the remaining 50\%). 
Depending on this outcome, participants either won or lost their selected bet stake. Cumulative winnings were not shown to minimize possible "wealth" effects (cf. Kahneman \& Tversky, 1979).

The probability of a win was fixed at chance with a win being completely independent of the bet stake placed by participants. Therefore, the expected value of each stake option was, on average, zero pence, thus meaning that there was no financial incentive for participants to favor either stake (other than any individual risk preferences whichas is standard-were assumed to be sufficiently stable over time and should not be influenced by preceding-trial outcome in the manner assessed here). With only two choice options available, participants were always aware of the outcome that would have ensued had they placed the alternative stake. We expected that such counterfactual information would bias future choice (e.g., Mellers, 1999).

Importantly, on two thirds of trials, participants were responsible for choosing the stake, with these trials providing the "agency" conditions (see Figure 1). Trials in which participants were not responsible for the bet placed (this being selected by the computer instead and then executed by the participant) constituted a "no-agency" condition that served to control for valence of outcome. Importantly, this allowed for neural correlates of agency-specific regret (i.e., worse outcome than from what would have arisen from the alternative bet choice) to be disambiguated from mere aversive outcome effects (i.e., such a loss regardless of agency). The no-agency trials, which comprised the remaining one third of trials on which participants were instructed to place the bet chosen by the computer, were randomly interleaved with the agency trials in an event-related design.

Participants each played five 8-min runs of the game, each including 120 trials. Participants were informed that the outcomes from a random selection of 100 trials, selected after the experiment, would determine their earnings for the entire experiment. Because participants did not know which trials would be selected, they were assumed to treat all trials as having an equal potential impact on their financial gain.

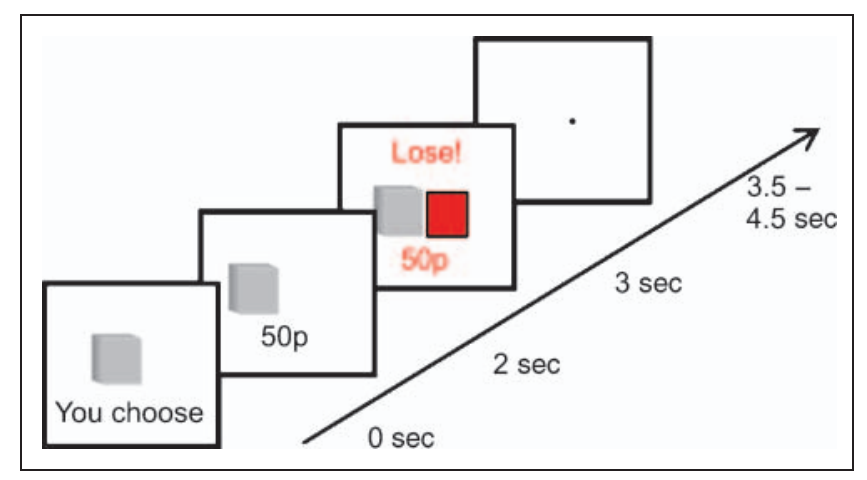

Figure 1. Schematic timeline of events within an illustrative agency trial, showing a cue for the participant to decide whether to place a $50 \mathrm{p}$ or $10 \mathrm{p}$ bet, followed by the outcome and finally a varied intertrial interval.
Our main behavioral-dependent measure comprised participants' trial-by-trial tendency to repeat at trial $t$ the bet placed at trial $t-1$. Actual wins and losses were randomized throughout, whereas the overall number of trials falling into the two stake levels was choice dependent. We also explored how any tendency to repeat the previous bet, contingent on the outcome of the previous trial, changed from early runs (1-3) to late runs (3-5) of the game. Finally, we acquired RT data. Analysis of current RT to select the bet (on agency trials) was conditionalized on the outcome of the previous trial (which could be an agency or no-agency trial).

\section{Imaging Acquisition}

We scanned participants in a 3-T Allegra scanner (Siemens, Erlangen, Germany) operated with its standard head transmit-receive coil. The manufacturer's standard automatic three-dimensional shim procedure was performed at the beginning of each experiment. Participants were scanned with a single-shot gradient-echo EPI sequence with the following imaging parameters: 40 oblique transverse slices, slice thickness $=2 \mathrm{~mm}$, gap between slices $=1 \mathrm{~mm}$, repetition time $=2.4 \mathrm{sec}, \alpha=90^{\circ}$, echo time $=30 \mathrm{msec}$, bandwidth $=3551 \mathrm{~Hz} /$ pixel, bandwidth in phase-encoding (PE) direction $=47.3 \mathrm{~Hz} /$ pixel, direction anterior-posterior field of view $=192 \times 192 \mathrm{~mm}^{2}$, matrix size $=64 \times 64$, fat suppression. BOLD sensitivity losses in the OFC and the amygdala because susceptibility artifacts were minimized by applying a $z$-shim gradient moment of $-0.4 \mathrm{mT} / \mathrm{m} / \mathrm{msec}$, a slice tilt of $-30^{\circ}$, and a positive PE gradient polarity (Weiskopf, Hutton, Josephs, \& Deichmann, 2006). EPI magnitude images were reconstructed from the complex $k$-space raw data using a generalized reconstruction method on the basis of the measured EPI $k$-space trajectory to minimize ghosting (Josephs, Deichmann, \& Turner, 2000). EPI data acquisition was monitored on-line using a real-time reconstruction and quality assurance system (Weiskopf et al., 2007). We acquired field maps for each subject at the start of scanning (Siemens standard double-echo gradient-echo field map sequence, echo time $=12.46 \mathrm{msec}$, repetition time $=10.2 \mathrm{msec}$, matrix size $=64 \times 64$, 64 slices covering the whole head, voxel size $=3 \times 3 \times 3 \mathrm{~mm}$ ). These allowed for calculation of static geometric distortions caused by susceptibility-induced field inhomogeneities, which were used to correct EPI images for these static distortions and any changes in these distortions because of head motion (Hutton et al., 2002; Andersson, Hutton, Ashburner, Turner, \& Friston, 2001). At the end of the scanning session, we acquired a T1-weighted anatomical scan for each participant using a modified driven equilibrium Fourier transform sequence (Ugurbil et al., 1993).

\section{Imaging Processing and Analysis}

Image preprocessing and data analysis were implemented using Statistical Parametric Mapping software in Matlab7.4 
(SPM5; Wellcome Trust Centre for Neuroimaging, University College London). After discarding the first six volumes of each run, to allow for T1 equilibration, EPI images were corrected for geometric distortions caused by susceptibilityinduced field inhomogeneities. Field maps were processed for each participant using the FieldMap toolbox implemented in SPM5 (Hutton, Deichmann, Turner, \& Andersson, 2004). The images were then realigned and unwarped using SPM5 (Andersson et al., 2001), which allows the measured static distortions to be included in the estimation of distortion changes associated with head motion. Each participant's structural image was then coregistered to the mean of the motion-corrected functional images using a 12-parameter affine transformation and segmented according to the standard procedure in SPM5 (Ashburner \& Friston, 2005). The spatial normalization parameters resulting from the previous step were then applied to the functional images to allow for intersubject analysis, and finally these images were smoothed using an 8-mm FWHM Gaussian kernel in accord with the standard SPM approach.

For each participant, we constructed two event-related general linear models (one to explore the response to the outcome of the current trial and a second testing trial-totrial effects). In the first model, eight regressors of interest were included to allow us to assess BOLD-signal response patterns to the eight outcome categories. These eight outcome categories were conditionalized on the outcome of the current trial, with the three orthogonal factors of agency or no agency, win or loss, and plus 50p or 10p stake. Given our short trial length (of 4 sec on average) we modeled trials as compound events, accounted for by one regressor onset per trial, at the time of outcome. These onsets were modeled by stick functions and then convolved with a canonical hemodynamic response function and its temporal derivative, at the time point at which participants received the outcome. Motion parameters defined by the realignment procedure were entered as six regressors of no interest, along with 17 additional regressors of cardiac phase (10 regressors), respiratory phase (6 regressors), and respiratory volume (1 regressor). We generated statistical parametric maps from our contrasts of interest, which included the main effects of win versus loss, high stake versus low stake, and agency versus no agency along with their interactions. The interaction of critical interest was between all three factors, specifically indicating increased or decreased activity for the regret-related outcome types relative to the relief-related outcome types on agency trials in particular. For this contrast, we were particularly interested in a priori ROIs within the striatum (including the caudate and putamen regularly implicated in both absolute and relative reward processing, e.g., Chua, Gonzalez, Taylor, Welsh, \& Liberzon, 2009; Chandrasekhar, Capra, Moore, Noussair, \& Berns, 2008; Liu et al., 2007; Coricelli et al., 2005; O'Doherty et al., 2004), where the critical interaction sought was for activity greater for $50 \mathrm{p}$ wins than for 50p losses but conversely greater for 10p losses than for $10 \mathrm{p}$ wins, specifically when participants were agents of the stake choice. Furthermore, on the basis of the proposed role of the OFC in regret (e.g., Coricelli et al., 2005; Camille et al., 2004), we predicted the inverse interaction to be represented in the OFC (anatomically defined using the WFUPickAtlas toolbox in SPM5). Within these ROIs, we report activity that is significant at a small volume, voxellevel, family-wise error (FWE) corrected threshold of $p<$ .05 . For regions where we had no a priori anatomical hypothesis, we report only activity surviving whole-brain cluster-level corrected significance at $p<.001$. We implemented group-level random-effects analysis using onesample $t$ tests on the contrast images obtained from each contrast of interest for each participant.

A second model for each participant separated the eight outcome-contingent regressors according to which bet stake was chosen on the subsequent trial, giving us 16 regressors of interest and allowing us to explore differences in outcome-related responses when participants then stick with the same choice versus switch to a different choice. Again outcome onsets were modeled with stick functions at the time of outcome, then convolved with a canonical hemodynamic response function and its temporal derivative. Because trials followed by a no-agency control trial (i.e., when there was no free choice on the subsequent trial) could not be categorized with respect to a later choice by the participant, these were included as a single regressor of no interest. We generated statistical parametric maps from our contrasts of interest. To assess the mechanism underlying the behavioral response to regret-related outcomes in our task, we tested the two-way interaction of ["stick" > "switch" after regret] > ["stick" > "switch" after relief], where losing 50p and winning 10p with agency were considered operationally as regret related, whereas winning 50p and losing 10p with agency were considered operationally as relief related. This contrast allowed us to explore the brain networks involved in a tendency to repeat previous regret-related choices more than previous relief-related choices (as recently documented in a purely behavioral study by Nicolle, in preparation). On the basis of a possible dorsal-ventral dissociation in the roles of the striatum in reward learning and goal-directed action (Wickens et al., 2007), we constructed ROIs within the left and right dorsal and ventral striatum. A hypothesized involvement of ACC in possible conflict monitoring, potentially arising because the above behavioral strategy would be in apparent conflict with the relatively decreased value of the regret-related outcome, also led us to test the same contrast within an anatomical ROI for bilateral ACC. The same statistical thresholds were used as described above.

\section{RESULTS}

Behaviorally, we found an increased tendency toward repeating previously regret-related choices (when having acted as an agent) relative to previously relief-related choices. This effect was found to reflect an early bias, 
which diminished significantly with increasing run number $($ stake $\times$ outcome $\times$ run $), F(4,72)=3.229, p<.05$. When the outcome was associated with no agency, neither the Stake $\times$ Outcome effect, $F(1,18)=2.896, p=n s$, nor its interaction with run number, $F(4,72)=0.567, p=n s$, was significant.

Figure 2A shows the probability of repeating the previous bet at trial $t$ contingent on each outcome associated with agency at $t-1$ in early and late runs. The early bias for repeating compared with switching from a previously regretrelated choice was a trend for regret-related 50p losses in Run $1, t(19)=1.830, p=.083$, and significant in Run 2, $t(19)=2.759, p<.02$. A tendency for participants to repeat $10 \mathrm{p}$ bets more than $50 \mathrm{p}$ bets was evident overall and was found to interact significantly with agency, $F(1,18)=$ $26.892, p<.001$, suggesting a general tendency for a riskaverse avoidance of the $50 \mathrm{p}$ bet when participants have agency in all but those trials that followed a 50p loss, where participants evidently preferred to repeat the $50 \mathrm{p}$ bet.

A further Run $\times$ Agency $\times$ Outcome interaction indicated a greater tendency to repeat bets after losses than after wins, but only when these outcomes were associated with agency, an effect which also decreased over time, $F(1$, $18)=2.914, p<.05$. One possible explanation for this effect might invoke the phenomenon of overalternation (or the gambler's fallacy), whereby the probability of a



Figure 2. The figure shows (A) an increased tendency to repeat, at trial $t$, the $50 \mathrm{p}$ bet after a regret-related $50 \mathrm{p}$ loss than after a reliefrelated $50 \mathrm{p}$ win at trial $t-1$. Choice behavior is shown for early and late runs in the task for trials associated with and without agency. (B) Quickened RTs (in msec) at trial $t$ after outcomes that would have been better from the alternative choice (regret at $t-1$ ) compared with outcomes that would have been worse from the alternative choice (relief at $t-1$ ), but only when trial $t-1$ was associated with agency. Error bars show the SEM. win is (mis)perceived to increase after a loss and decrease after a win (e.g., see Croson \& Sundali, 2005). However, such an explanation alone would not predict the difference we observed between agency and no-agency conditions. In addition, there was no evidence of an enhanced tendency to bet 50p more after losses than after wins because a function of an increase in the number of times a loss is experienced in a sequence of bets, $F(1,18)=0.89, p=$ $n s$. Thus, the behavioral pattern of results was not explicable solely by an overalternation fallacy.

We also found a significant RT effect (for RT to place the next agency bet) with participants having significantly quicker RTs after regret-related outcomes (that were obtained with agency) compared with relief-related outcomes (also with agency), $t(19)=2.868, p<.01$, or compared with the equivalent outcomes with no agency, $t(19)=2.159$, $p<.05$. Speeded RTs in response to regret (Figure 2B) accord with some previous results (Chua et al., 2009).

\section{fMRI Main Effects}

Our fMRI main effects are shown in Table 1. Increased activity for wins compared with losses overall was seen in bilateral ventral striatum (whole-brain FWE corrected at $p<$ .05; see SPM in Figure 3A). This pattern of increased activity was also significantly greater for $50 \mathrm{p}$ wins relative to $10 \mathrm{p}$ wins bilaterally (small-volume FWE corrected at $p<.05$ in the whole striatum). This finding is consistent with previous reports of striatal responses to rewards compared with losses (Tom, Fox, Trepel, \& Poldrack, 2007; O’Doherty et al., 2004; Delgado, Nystrom, Fissell, Noll, \& Fiez, 2000). Although we found no significant interaction of this response with agency, indicating that the overall response to wins was not dependent on being responsible for the choice. No areas were significantly more active for all losses compared with all wins overall. For completeness, the main effects of agency and of stake are presented in Table 1.

\section{Activity Reflecting What Might Have Been}

A significant Stake $\times$ Outcome interaction reflected increased activity in bilateral ventral striatum for the two counterfactual outcomes where an outcome could have been worse (i.e., winning 50p and losing 10p) relative to when outcomes could have been better (i.e., losing 50p and winning 10p) at $p<.05$ FWE corrected for the whole striatum (see Figure 3B). This finding is consistent with the expression of a counterfactual signal in bilateral striatum, as reported previously (Chua et al., 2009; Chandrasekhar et al., 2008; Coricelli et al., 2005). The peak activity for this effect was slightly more anterior and dorsolateral within the putamen, compared to the main effect of wins. In a region of left putamen, this Stake $\times$ Outcome interplay was further dependent on having choice responsibility, that is, agency (with the three-way interaction surviving FWE correction at $p<.05$ when using a functional ROI taken from the orthogonal two-way interaction), thus reflecting 
Table 1. The Table Shows Significant Activation for the Main Effects of Outcome, Stake, and Agency at the Time of Outcome

\begin{tabular}{|c|c|c|c|}
\hline Brain Regions & MNI Coordinates of Local Maxima & Voxel Number & Voxel $t$ Score \\
\hline \multicolumn{4}{|l|}{ Win $>$ Loss } \\
\hline R putamen & $15,12,-3$ & 91 & 7.50 \\
\hline L middle frontal (BA 10) & $-36,54,6$ & 141 & 6.82 \\
\hline Bilateral lingual sulcus (BA 30) & $3,-72,3$ & 279 & 5.70 \\
\hline L insula (BA 13) & $-27,18,3$ & 32 & 5.40 \\
\hline L caudate & $-12,12,0$ & 31 & 4.38 \\
\hline \multicolumn{4}{|l|}{$50 p>10 p$} \\
\hline $\mathrm{R}$ anterior cingulate (BA 24) & $6,24,15$ & 36 & 6.50 \\
\hline L substantia nigra & $-3,-12,-12$ & 57 & 5.25 \\
\hline $\mathrm{R}$ anterior cingulate (BA 10) & $3,51,9$ & 152 & 4.89 \\
\hline L inferior frontal (BA 47) & $-42,24,-15$ & 29 & 4.77 \\
\hline R caudate & $12,3,3$ & 12 & 4.12 \\
\hline \multicolumn{4}{|l|}{$10 p>50 p$} \\
\hline R parahippocampal gyrus & $24,-18,-18$ & 38 & 6.16 \\
\hline \multicolumn{4}{|l|}{ Agency $>$ No Agency } \\
\hline R insula (BA 13) & $33,18,6$ & 15 & 4.27 \\
\hline \multicolumn{4}{|l|}{ No Agency > Agency } \\
\hline Bilateral precuneus (BA 7) & $3,-60,39$ & 1471 & 11.70 \\
\hline $\mathrm{R}$ middle temporal gyrus & $48,-54,18$ & 815 & 8.90 \\
\hline L middle temporal gyrus (BA 39) & $-45,-60,21$ & a & 6.67 \\
\hline Bilateral lingual sulcus (BA 18) & $-6,-78,-6$ & 200 & 6.58 \\
\hline R middle temporal gyrus (BA 21) & $66,-12,-12$ & 100 & 6.27 \\
\hline R medial frontal gyrus (BA 9) & $12,51,30$ & 48 & 6.20 \\
\hline L middle frontal gyrus (BA 6) & $-42,6,48$ & 39 & 5.93 \\
\hline R hippocampus & $30,-21,-18$ & 58 & 5.70 \\
\hline $\mathrm{L}$ rectus gyrus (BA 11) & $-3,42,-21$ & 138 & 5.62 \\
\hline
\end{tabular}

Note that no significant voxels were found for the main effect of loss $>$ win.

Clusters are reported at a voxel-level significance threshold of $p<.001$ uncorrected with an extent of $>10$ voxels.

${ }^{\mathrm{a}}$ Part of the bilateral precuneus cluster.

a relief $>$ regret difference (see Figure 3C). A corresponding cluster in the right putamen showed a similar effect at a lower significance level $(p<.002$ uncorrected, mentioned here as it points to there being no hemispheric differences in this effect). We found no regions where activity increased during the outcomes that could have been better versus worse or showing such a pattern that interacted with agency (i.e., showing putative regret $>$ relief effects), even within our anatomically defined ROIs, including those encompassing the whole OFC.

\section{Dorsal Striatum Reflects the Behavioral Tendency to Repeat Regret-related 50p Losses}

To address what drives participants to repeat a regretrelated gamble choice (i.e., stake) on the immediately following trial, we divided outcomes into those where participants chose to repeat the same choice on the subsequent trial ("stick" trials) and those where they switched to the alternative gamble choice ("switch" trials). Activity in left dorsal putamen (Figure 4) was greater when participants 


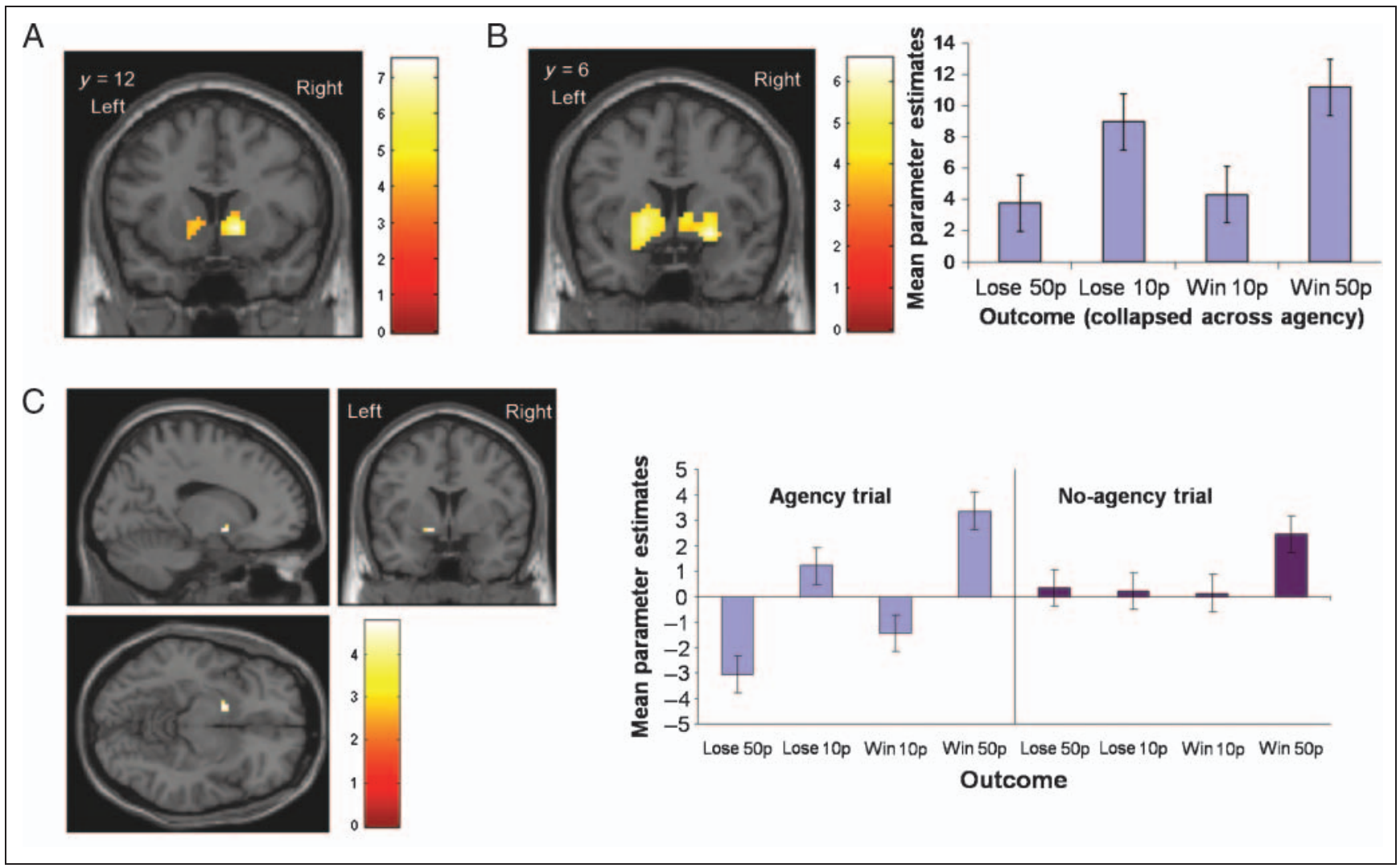

Figure 3. Group SPM data, thresholded at $p<.001$ for display purposes, shown on a normalized canonical template brain. (A) Activity associated with overall Win $>$ Loss. (B) Activity associated with counterfactual context, where (50p bet $>10 \mathrm{p}$ bet) win $>$ (50p bet $>10 \mathrm{p}$ bet) loss. The upper right plot shows the mean beta values for the four outcome types (collapsed across agency) in the peak voxel at $24,6,-9$, with absolute outcome value increasing left to right on the $x$-axis. (C) SPM for the three-way interaction under which the Stake $\times$ Outcome interplay (as per B) was greater for agency compared with no-agency trials. The lower right plots show the mean beta values for the eight trial types in the peak voxel at $-15,3,-9$. Error bars show the SEM. Coordinates are in MNI space.

subsequently repeated a preceding regret-related choice (small-volume FWE corrected $p<.05$ ) but showed no significant difference between choices to stick versus switch after relief-related choices (Montreal Neurological Institute $[\mathrm{MNI}]$ peak $-24,9,-3)$. We did not find any region with significantly increased activity when participants chose to switch to the alternative bet after a regret-related outcome. Furthermore, no regions significantly reflected choice following outcomes associated with no agency. We found that activity in right dorsal striatum during $50 \mathrm{p}$ losses with
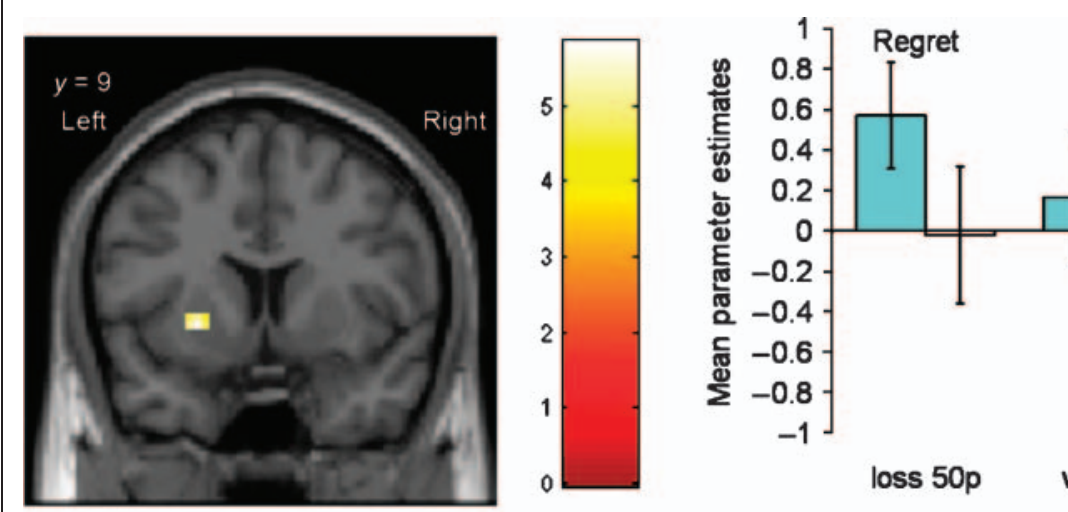

loss 50p win 10p win 50p

Outcome at $t$ with agency

Figure 4. Group SPM data thresholded at $p<.001$ for display purposes and shown on a normalized canonical template brain, showing activity for agency-related outcomes. The plot shows activity in left putamen for the contrast (Stick $>$ switch after regret) $>$ (Stick $>$ switch after relief), with the mean beta values in the peak voxel for the left putamen at $-24,9,-3$, shown for all outcome types with agency when the following choice was to stick or switch. Error bars show the SEM. Coordinates are in MNI space. 
agency showed a significant linear decrease from early to late runs relative to activity during 50p wins with agency (small-volume FWE corrected in the whole striatum, $p<$ $.05)$. This may reflect the increased behavioral tendency to repeat regret-related 50p losses in early runs (cf. Figure 2A).

Activity in the ACC (MNI 3, 33, 24) also showed a significant interaction of choice and previous outcome (smallvolume FWE corrected $p<.05$ ), apparent in the same contrast that had revealed the left dorsal putamen response. This region showed increased activity associated with the subsequent choice to "stick" with a previously regret-related bet and decreased activity associated with the subsequent choice to "stick" with a previously reliefrelated bet. Activity here did not differentiate regret- and relief-related outcomes when it came to decisions to "switch" on the next trial.

\section{DISCUSSION}

A common assumption in theoretical discussions of regret is that it is a highly aversive emotion that motivates choices to avoid its future re-occurrence. However, there can be other consequences of regret. For example, Connolly and Zeelenberg (2002) suggest that a common response to regret (including anticipated regret) is to make more "normal" decisions that deviate less from a norm or status quo and thereby help an individual feel their decisions are more justified. Zeelenberg (1998) showed that, compared with disappointment, regret encourages a desire to make up for a mistake and allow a second chance. In a conceptually similar suggestion, Loftus and Loftus (1983) propose that "chasing" behavior in response to near misses provides gamblers with the opportunity to eliminate the impact of regret by potentially making up for their mistakes. In our iterative gambling task under uncertainty, we found that healthy participants show a clear behavioral tendency to repeat a previously regret-related choice during early runs (see Figure 2), a pattern of behavior we might term regretrelated chasing because of its analogy with the chasing behavior shown by some problem gamblers (Reid, 1986). One attempt to explain such behavior might invoke the gambler's fallacy, whereby the probability of a win is (mis)perceived to increase after the experience of a loss and decrease after a win (e.g., see Croson \& Sundali, 2005), thus encouraging a higher risk bet after a loss. It is conceivable that such an effect might diminish over time (as for the behavioral pattern here) if participants estimated the outcome probabilities with more precision over time. However, the gambler's fallacy alone would not explain the differences we observed under conditions of agency versus no agency nor the interaction with stake whereby a differential tendency to repeatedly bet 50p after regret is greater between 50 p than between 10p outcomes. Furthermore, an analysis of sequences of identical outcomes did not reveal any significant tendency to place a 50p bet more after losses than after wins when more losses were experienced in a sequence, although that would be expected from the gambler's fallacy.

We have argued that the observed regret-related chasing may provide a means of regulating current feelings of regret by allowing the possibility of "making up" for mistakes, in contrast to standard theories of regret (c.f. Mellers et al., 1997; Bell, 1982; Loomes \& Sugden, 1982; Savage, 1951). Alternatively, repetition of a previously regret-related outcome may reflect decision inertia, which some suggest is a strategy allowing one to regulate regret (e.g., Inman \& Zeelenberg, 2002; Ritov \& Baron, 1990; Kahneman \& Tversky, 1982). Our study suggests possible differences in the impact of anticipated and experienced regret on future choice. A novel aspect of our task is that it allowed us to explore outcome-contingent choices (i.e., in response to experienced regret- or relief-related outcomes) under a state of ambiguity, whereas previous studies of regret have assessed choices in the presence of anticipated risk (e.g., Mellers, 1999). Real-life decisions are often made in a state of ambiguity or uncertainty about outcome probabilities. Ellsberg (1961) proposed that such decisions are treated differently from decisions under risk, and fMRI studies have recently also shown the two to be neuronally dissociated (e.g., Bach, Seymour, \& Dolan, 2009). Thus, although anticipated risk of regret may encourage avoidance of regret-related options, regret experienced under ambiguity (as here) may lead to choices that appear driven by a motivation to compensate for an apparent mistake.

We investigated brain mechanisms that underlie regretrelated choice repetition when participants were responsible for their choice (as contrasted with the no-agency conditions). We found increased activity in the dorsal striatum when subjects made such choices. Although we did not find OFC involvement in regret-related outcomes, this might reflect some lack of sensitivity because of our inability to explore regret as a parametric variable, unlike previous studies (cf. Coricelli et al., 2005). Indeed, one might also have expected greater OFC involvement to correlate with a tendency toward increased regret aversion over time. However, this was not the behavioral tendency we observed, which might also contribute to a lack of sensitivity in isolating any OFC involvement. As our task explored outcome-contingent choice under ambiguity, this seems likely to be the dominant factor in accounting for differences in the present results versus previous studies, as the latter tended to explore choice under a state of anticipated risk of regret (e.g., Mellers et al., 1997).

Several previous fMRI studies have found the striatum to be important for the processing of primary rewards (O’Doherty, Deichmann, Critchley, \& Dolan, 2002) as well as for more abstract rewards, including money (Delgado et al., 2000), romantic love (Aron et al., 2005), and humor (Mobbs, Greicius, Abdel-Azim, Menon, \& Reiss, 2003). The striatum is also implicated in the encoding of action-outcome contingencies (Tanaka, Balleine, \& O’Doherty, 2008; Delgado, Miller, Inati, \& Phelps, 2005). Other studies have implicated striatal circuits in processing of rewards relative 
to a counterfactual reference point, as is central to the phenomena of relief/rejoicing (Chua et al., 2009; Chandrasekhar et al., 2008; Liu et al., 2007; Coricelli et al., 2005). Our finding of relatively increased activity in ventral striatum during relief-related outcomes (i.e., those that would have been worse if a different choice had been made) is consistent with this role in processing rewards that are relative to a counterfactual reference point. Furthermore, we find a critical role for agency in this pattern of activity, thus contributing to an ongoing debate as to the role of responsibility in regret and relief.

The striatum has been implicated not only in the passive receipt of rewards (O'Doherty et al., 2004) but also in encoding violations of expectations in the form of prediction error signals, which reinforcement learning models show to be central to guiding future behavior (Berns, McClure, Pagnoni, \& Montague, 2001; Sutton \& Barto, 1998). Moreover, evidence of putative positive fictive prediction error signals in the striatum, which can update the value of actions not taken, suggests that counterfactual reference points may also be important for guiding of behavior in this way (Lohrenz, McCabe, Camerer, \& Montague, 2007). Our present study has found that value differences between an obtained outcome and that which might have been obtained (had a different choice been taken) are also represented in ventral striatum. This indicates a striatal role in between-option counterfactual comparisons, as well as within-option comparisons while also demonstrating the critical role of agency in this process.

If the outcome received from a particular action violates our predictions, the resulting error signal will incrementally modify the value of repeating this action in the future. Dopaminergic neurons that project from the ventral tegmental area to the ventral striatum are critical to reward prediction error signals in the brain (Pessiglione, Seymour, Flandin, Dolan, \& Frith, 2006; Schultz, Dayan, \& Montague, 1997). These neurons show increased firing rate when outcomes are better than predicted and dips when they are worse than predicted. Through functional imaging, Seymour, Daw, Dayan, Singer, and Dolan (2007) found both appetitive and aversive prediction errors to be reflected in increased activity in the ventral striatum. This finding raises the possibility that increased striatal activity associated with regret-related choice repetition may reflect an aversive prediction error (or fictive prediction error) signal at the time of the regretful outcome. However, we would argue here that this would not account for the increased tendency to repeat the regret-related choice because such aversive prediction error signals should predict decreased value for repeating such actions in the future. Indeed, it is important to note that the observed tendency to repeat previously regret-related bets more than previously relief-related bets is contrary to expectations from traditional learning models that update the value of a choice, as a function of either standard or regret-based (or fictive) prediction errors (e.g., Marchiori \& Warglien, 2008; Lohrenz et al., 2007; Rescorla \& Wagner, 1972). On such accounts, the value of a choice option should be incrementally decreased for a negative or regretful outcome. Such learning models thus provide a poor fit to the choice behavior we observe.

We found activity in ventral striatum reflecting the value of an experienced outcome relative to what might have been under a different choice (Figure 3C). However, activity in dorsal striatum was particularly involved in regretrelated choice repetition (Figure 4), indicating regional specialization in striatal involvement for this task. This aspect of our findings is in general accord with previous work showing the dorsal striatum to be especially important in stimulus-response-reward learning, whereas the ventral striatum is important for stimulus-reward prediction (Delgado et al., 2005; O'Doherty et al., 2004; Tricomi, Delgado, \& Fiez, 2004). O’Doherty et al. (2004) proposed that the ventral and dorsal striatum play dissociable roles in the control of our action, with the former serving a "critic" role important for passively predicting the value of future states, while the latter serves an "actor" role involved in updating stimulus-response-reward associations and reinforcing or gating the selection of future high value actions. Dopaminergic projections to the dorsal and ventral striatum originate from different sources (from the substantia nigra and the ventral tegmental area, respectively), with possible associated differences in the temporal properties of dopaminergic innervation, which may provide a physiological basis for their different roles in behavior (see review by Wickens et al., 2007). Here our findings add a new line of support for such a functional dissociation along the dorsal-ventral axis of the striatum while also showing for the first time such dissociation in a context where rewards are relative to their counterfactual alternatives.

Along with outcome-induced updating of action-reward contingencies, activity in dorsal striatum has also been found to reflect choice-induced modifications of value. For example, Sharot, De Martino, and Dolan (2009) found increased caudate activity associated with postchoice increases in the subjective value of the option participants had recently chosen. They proposed that this increased activity may be associated with a desire to reduce cognitive dissonance. Their findings suggest a role for dorsal striatum in higher order, temporally delayed, goal-directed action as well as in simpler examples of stimulus-response-reward learning. Similarly, we argue here that our findings may reflect a role of the dorsal striatum in updating the subjective value of repeating a previously regret-related choice, perhaps motivated by a desire to make up for our mistakes and defend the justifiability of our past choices, both of which are associated with a reduction of cognitive dissonance (Roese \& Summerville, 2005). Tanaka et al. (2004, 2007) have found the dorsal striatum to be active when choosing larger delayed rewards in favor of smaller immediate rewards, supporting its role in the motivation of actions toward longer term goals, whereas more ventral regions of the striatum were active when choices were more impulsive, that is, in favor of the smaller immediate rewards. 
We questioned whether the value-related signal, observed in ventral striatum, may be regulated or modified by adherence to higher order behavioral goals. There was no evidence that decreased ventral striatal activity to regretrelated outcomes was influenced by a choice to stick or switch on the next trial while dorsal striatum did show such an effect. Anatomical studies show ascending spirals within both a striato-nigro-striatal loop and a limbic-to-motor striatocortical loop, with the direction of both being ventromedial to dorsolateral (e.g., Haber \& Knutson, 2009; Draganski et al., 2008; Haber, Fudge, \& McFarland, 2000) and no evidence of direct information transmission from dorsal to ventral striatum. It is possible, however, that interactions between processing in ventral and dorsal striatum are expressed elsewhere. The ACC, with projections to ventral and dorsal striatum, is a prime candidate for this. Indeed, we found responses in ACC that reflected a decision to repeat previously regret- and relief-related bets. Because ACC is often implicated in the monitoring of conflict (e.g., Botvinick et al., 2001), the observed ACC activity here may be associated with monitoring of conflict between value and choice. Another possibility, however, is that it regulates or gates value-related activity in ventral striatum, thereby facilitating choice-related activity in the dorsal striatum.

Our findings have potential implications for understanding the chasing behavior exhibited by some problem gamblers. In a large sample of horse races, gamblers tended to go for long shot gambles (low probability of high possible gain) on the last race of the day, as if trying to break even or to make right earlier losses (McGlothlin, 1956). Losing gamblers also tend to increase their bets more than did winning gamblers. This chasing behavior might in principle arise despite problem gamblers potentially being able to recognize the consequences of their choices, to anticipate future regret, or to assign self-blame. Instead, chasing may provide gamblers with the opportunity to modulate current regret by trying to make up for their mistakes - although such an incentive may then diminish subjective and neuronal sensitivity to the possibility of a further loss or regretful event. The present data suggest that regret-induced chasing may be generally pervasive. Evidence for a link between compulsive gambling and dopamine agonist treatment of Parkinson patients (Molina et al., 2000) supports the idea that dopaminergic projections to striatum may play a critical role. Finally, the experience of "near miss" outcomes in gambling has been proposed as having a similar conditioning effect on future behavior as experiencing a full win (Reid, 1986) while also being associated with strong feelings of regret because of the relative ease with which the better counterfactual alternative outcome of a full win can be brought to mind (Kahneman \& Tversky, 1982). Near misses have recently been associated with increased striatal activity as well as with a simultaneously increased desire to continue gambling, despite these outcomes being rated as more unpleasant than full misses (Clark, Lawrence, AstleyJones, \& Gray, 2009).
Here, we show that operationalized regret, under certain choice constraints, can lead to choices that appear to reflect attempts to make up for apparent mistakes and, in so doing, drive subsequent chasing behavior. Furthermore, our findings suggest a central role for the striatum in driving this behavior in a manner that accords with current models of dorsal-ventral dissociation for striatal function. Further consideration of the role of regret- regulatory strategies in chasing behavior, along with the neuronal mechanisms involved, is likely to be crucial in understanding mechanisms driving maladaptive behaviors such as gambling as well as that seen in patient populations where compulsive gambling can sometimes be a side effect of neuromodulatory therapy, as seen in Parkinson's disease.

\section{Acknowledgments}

This study was completed under a Wellcome Trust program grant to R. J. D., a Brain Research Trust prize studentship to A. N., and a personal grant to D. R. B. from the Swiss National Science Foundation. J. D. is a Royal Society 2010 Anniversary Research Professor.

Reprint requests should be sent to Antoinette Nicolle, Wellcome Trust Centre for Neuroimaging, 12 Queen Square, London, WC1N 3BG, United Kingdom, or via e-mail: a.nicolle@fil.ion.ucl.ac.uk.

\section{REFERENCES}

Andersson, J. L., Hutton, C., Ashburner, J., Turner, R., \& Friston, K. (2001). Modelling geometric deformations in EPI time series. Neuroimage, 13, 903-919.

Aron, A., Fisher, H., Mashek, D. J., Strong, G., Li, H., \& Brown, L. L. (2005). Reward, motivation, and emotion systems associated with early-stage intense romantic love. Journal of Neurophysiology, 94, 327-337.

Ashburner, J., \& Friston, K. J. (2005). Unified segmentation. Neuroimage, 26, 839-851.

Bach, D. R., Seymour, B., \& Dolan, R. J. (2009). Neural activity associated with the passive prediction of ambiguity and risk for aversive events. Journal of Neuroscience, 29, $1648-1656$.

Bell, D. E. (1982). Regret in decision-making under uncertainty. Operations Research, 30, 961-981.

Berns, G. S., McClure, S. M., Pagnoni, G., \& Montague, P. R. (2001). Predictability modulates human brain response to reward. Journal of Neuroscience, 21, 2793-2798.

Botvinick, M., Braver, T., Barch, D., Carter, C., \& Cohen, J. (2001). Conflict monitoring and cognitive control. Psychological Review, 108, 624-652.

Bush, G., Vogt, B. A., Holmes, J., Dale, A. M., Greve, D., Jenike, M. A., et al. (2002). Dorsal anterior cingulate cortex: A role in reward-based decision making. Proceedings of the National Academy of Sciences, U.S.A., 99, 523-528.

Camille, N., Coricelli, G., Sallet, J., Pradat-Diehl, P., Duhamel, J., \& Sirigu, A. (2004). The involvement of the orbitofrontal cortex in the experience of regret. Science, 304, 1167-1170.

Chandrasekhar, P. V. S., Capra, C. M., Moore, S., Noussair, C., \& Berns, G. S. (2008). Neurobiological regret and rejoice functions for aversive outcomes. Neuroimage, 39, 1472-1484.

Chua, H. F., Gonzalez, R. T., Taylor, S. F., Welsh, R. C., \& Liberzon, I. (2009). Decision-related loss: Regret and disappointment. Neuroimage, 47, 2031-2040. 
Clark, L., Lawrence, A. J., Astley-Jones, F., \& Gray, N. (2009). Gambling near-misses enhance motivation to gamble and recruit win-related brain circuitry. Neuron, 61, 481-490.

Connolly, T. (1997). Regret and responsibility in the evaluation of decision outcomes. Organizational Behavior and Human Decision Processes, 70, 73-85.

Connolly, T., \& Zeelenberg, M. (2002). Regret in decision making. Current Directions in Psychological Science, 11, 212-216.

Coricelli, G., Critchley, H. D., Joffily, M., O’Doherty, J. P., Sirigu, A., \& Dolan, R. J. (2005). Regret and its avoidance: A neuroimaging study of choice behavior. Nature Neuroscience, 8, 1255-1262.

Critchley, H. D., Mathias, C. J., Josephs, O., O’Doherty, J., Zanini, S., Dewar, B., et al. (2003). Human cingulate cortex and autonomic control: Converging neuroimaging and clinical evidence. Brain, 126, 2139-2152.

Croson, R., \& Sundali, J. (2005). The gambler's fallacy and the hot hand: Empirical data from casinos. Journal of Risk and Uncertainty, 30, 195-209.

Delgado, M. R., Miller, M. M., Inati, S., \& Phelps, E. A. (2005). An fMRI study of reward-related probability learning. Neuroimage, 24, 862-873.

Delgado, M. R., Nystrom, L. E., Fissell, K., Noll, D. C., \& Fiez, J. A. (2000). Tracking the hemodynamic responses for reward and punishment in the striatum. Journal of Neurophysiology, 84, 3072-3077.

Draganski, B., Kherif, F., Kloppel, S., Cook, P. A., Alexander, D. C., Parker, G. J., et al. (2008). Evidence for segregated and integrative connectivity patterns in the human basal ganglia. Journal of Neuroscience, 28, 7143.

Ellsberg, D. (1961). Risk, ambiguity and the Savage axioms. Quarterly Journal of Economics, 75, 643-669.

Haber, S. N., Fudge, J. L., \& McFarland, N. R. (2000). Striatonigrostriatal pathways in primates form an ascending spiral from the shell to the dorsolateral striatum. Journal of Neuroscience, 20, 2369.

Haber, S. N., \& Knutson, B. (2009). The reward circuit: Linking primate anatomy and human imaging. Neuropsychopharmacology, 35, 4-26.

Hutton, C., Bork, A., Josephs, O., Deichmann, R., Ashburner, J., \& Turner, R. (2002). Image distortion correction in fMRI: A quantitative evaluation. Neuroimage, 16, 217-240.

Hutton, C., Deichmann, R., Turner, R., \& Andersson, J. L. R. (2004). Combined correction for geometric distortion and its interaction with head motion in fMRI. In Proceedings of ISMRM (Vol. 12). Kyoto, Japan.

Inman, J., \& Zeelenberg, M. (2002). Regret in repeat purchase versus switching decisions: The attenuating role of decision justifiability. Journal of Consumer Research, 29, 116-128.

Josephs, O., Deichmann, R., \& Turner, R. (2000). Trajectory measurement and generalised reconstruction in rectilinear EPI. In Proceedings of the 8th ISMRM (p. 1517). Denver, CO.

Kahneman, D., \& Tversky, A. (1979). Prospect theory-Analysis of decision under risk. Econometrica, 47, 263-291.

Kahneman, D., \& Tversky, A. (1982). The simulation heuristic. In D. Kahneman, P. Slovic, \& A. Tversky (Eds.), Judgment under uncertainty: Heuristics and biases (pp. 201-210). Cambridge, UK: Cambridge University Press.

Lesieur, H. R. (1984). The chase: Career of the compulsive gambler. Cambridge, MA: Schenkman.

Liu, X., Powell, D. K., Wang, H., Gold, B. T., Corbly, C. R., \& Joseph, J. E. (2007). Functional dissociation in frontal and striatal areas for processing of positive and negative reward information. The Journal of Neuroscience, 27, 4587-4597.

Loftus, G. R., \& Loftus, E. K. (1983). Mind at play. New York: Basic Books.
Lohrenz, T., McCabe, K., Camerer, C. F., \& Montague, P. R. (2007). Neural signature of fictive learning signals in a sequential investment task. Proceedings of the National Academy of Sciences, U.S.A., 104, 9493-9498.

Loomes, G., \& Sugden, R. (1982). Regret theory-An alternative theory of rational choice under uncertainty. Economic Journal, 92, 805-824.

Marchiori, D., \& Warglien, M. (2008). Predicting human interactive learning by regret-driven neural networks. Science, 319, 1111.

Markman, K. D., Gavanski, I., Sherman, S. J., \& Mcmullen, M. N. (1993). The mental simulation of better and worse possible worlds. Journal of Experimental Social Psychology, 29, 87-109.

McGlothlin, W. H. (1956). Stability of choices among uncertain alternatives. American Journal of Psychology, 69, 604-615.

Mellers, B. A. (1999). Emotion-based choice. Journal of Experimental Psychology: General, 128, 332-345.

Mellers, B. A., Schwartz, A., Ho, K., \& Ritov, I. (1997). Decision affect theory: Emotional reactions to the outcomes of risky options. Psychological Science, 8, 423-429.

Mobbs, D., Greicius, M. D., Abdel-Azim, E., Menon, V., \& Reiss, A. L. (2003). Humor modulates the mesolimbic reward centers. Neuron, 40, 1041-1048.

Molina, J. A., Sainz-Artiga, M. J., Fraile, A., Jiminez-Jiminez, F. J., Villanueva, C., Orti-Pareja, M., et al. (2000). Pathological gambling in Parkinson's disease: A behavioral manifestation of pharmacologic treatment? Movement Disorders, 15 , 869-872.

Nicolle, A. (in preparation). When there's no-one to blame but yourself: The role of regret and responsibility in decision-making. Thesis.

O’Doherty, J., Dayan, P., Schultz, J., Deichmann, R., Friston, K., \& Dolan, R. J. (2004). Dissociable roles of ventral and dorsal striatum in instrumental conditioning. Science, 304, 452-454.

O’Doherty, J., Deichmann, R., Critchley, H. D., \& Dolan, R. J. (2002). Neural responses during anticipation of a primary taste reward. Neuron, 33, 815-826.

Ordonez, L., \& Connolly, T. (2000). Regret and responsibility: A reply to Zeelenberg et al. (1998). Organizational Behavior and Human Decision Processes, 81, 132-142.

Pessiglione, M., Seymour, B., Flandin, G., Dolan, R. J., \& Frith, C. D. (2006). Dopamine-dependent prediction errors underpin reward-seeking behaviour in humans. Nature, 442, 1042-1045.

Reid, R. L. (1986). The psychology of the near miss. Journal of Gambling Behavior, 2, 32-39.

Rescorla, R. A., \& Wagner, A. R. (1972). A theory of Pavlovian conditioning: Variations in the effectiveness of reinforcement and non-reinforcement. In A. H. Black \& W. F. Prokasy (Eds.), Classical conditioning II: Current research and theory (pp. 64-69). New York: Appleton-Century-Crofts.

Ritov, I., \& Baron, J. (1990). Reluctance to vaccinate: Omission bias and ambiguity. Journal of Behavioral Decision Making, 3, 263-277.

Roese, N. J. (1994). The functional basis of counterfactual thinking. Journal of Personality and Social Psychology, 66, 805.

Roese, N. J., \& Olson, J. M. (2007). Better, stronger, faster self-serving judgment, affect regulation, and the optimal vigilance hypothesis. Perspectives on Psychological Science, 2, 124-141.

Roese, N. J., \& Summerville, A. (2005). What we regret most ... and why. Personality and Social Psychology Bulletin, 31, $1273-1285$.

Savage, L. (1951). The theory of statistical decision. Journal of the American Statistical Association, 46, 55-67. 
Schultz, W., Dayan, P., \& Montague, P. R. (1997). A neural substrate of prediction and reward. Science, 275, 1593-1599.

Seymour, B., Daw, N., Dayan, P., Singer, T., \& Dolan, R. J. (2007). Differential encoding of losses and gains in the human striatum. Journal of Neuroscience, 27, 4826-4831.

Sharot, T., De Martino, B., \& Dolan, R. J. (2009). How choice reveals and shapes expected hedonic outcome. Journal of Neuroscience, 29, 3760-3765.

Sutton, R., \& Barto, A. (1998). Reinforcement learning: An introduction. Cambridge, MA: MIT Press.

Tanaka, S. C., Balleine, B. W., \& O’Doherty, J. P. (2008). Calculating consequences: Brain systems that encode the causal effects of actions. Journal of Neuroscience, 28, 6750.

Tanaka, S. C., Doya, K., Okada, G., Ueda, K., Okamoto, Y., \& Yamawaki, S. (2004). Prediction of immediate and future rewards differentially recruits cortico-basal ganglia loops. Nature Neuroscience, 7, 887-893.

Tanaka, S. C., Schweighofer, N., Asahi, S., Sishida, K., Okamoto, Y., Yamawaki, S., et al. (2007). Serotonin differentially regulates short-and long-term prediction of rewards in the ventral and dorsal striatum. PLOS ONE, 2, e1333.

Tom, S. M., Fox, C. R., Trepel, C., \& Poldrack, R. A. (2007). The neural basis of loss aversion in decision-making under risk. Science, 315, 515-518.

Tricomi, E. M., Delgado, M. R., \& Fiez, J. A. (2004). Modulation of caudate activity by action contingency. Neuron, 41, 281-292.
Ugurbil, K., Garwood, M., Ellermann, J., Hendrich, K., Hinke, R. $\mathrm{Hu}, \mathrm{X}$., et al. (1993). Imaging at high magnetic fields: Initial experiences at 4T. Magnetic Resonance Quarterly, 9, 259-277.

Weiskopf, N., Hutton, C., Josephs, O., \& Deichmann, R. (2006). Optimal EPI parameters for reduction of susceptibilityinduced BOLD sensitivity losses: A whole-brain analysis at $3 \mathrm{~T}$ and $1.5 \mathrm{~T}$. Neuroimage, 33, 493-504.

Weiskopf, N., Sitaram, R., Josephs, O., Veit, R., Scharnowski, F., Goebel, R., et al. (2007). Real-time functional magnetic resonance imaging: Methods and applications. Magnetic Resonance Imaging, 25, 989-1003.

Wickens, J. R., Budd, C. S., Hyland, B. I., \& Arbuthnott, G. W. (2007). Striatal contributions to reward and decision making. Making sense of regional variations in a reiterated processing matrix. Annals of the New York Academy of Sciences, 1104, 192-212.

Zeelenberg, M. (1998). Reconsidering the relation between regret and responsibility. Organizational Behavior and Human Decision Processes, 74, 254-272.

Zeelenberg, M. (2000). Regret and responsibility resolved? Evaluating Ordonez and Connolly's (2000) conclusions. Organizational Behavior and Human Decision Processes, 81, 143-154.

Zeelenberg, M., \& Pieters, R. (2007). A theory of regret regulation 1.0. Journal of Consumer Psychology, 17, 3-18.

Zeelenberg, M., van Dijk, W. W., Manstead, A. S. R., \& van der Pligt, J. (1998). The experience of regret and disappointment. Cognition and Emotion, 12, 221-230. 